\title{
Perfil dos praticantes de beach tennis no município de Criciúma
}

\author{
Profile of beach tennis players in the city of Criciúma \\ Perfil de los practicantes de tenis playa en la ciudad de Criciúma
}

Recebido: 30/11/2021 | Revisado: 06/12/2021 | Aceito: 19/12/2021 | Publicado: 24/12/2021

\author{
Thainá Ribeiro da Rosa \\ ORCID: https://orcid.org/0000-0003-0287-4089 \\ Universidade do Extremo Sul Catarinense, Brasil \\ E-mail: thainaribeiroo100@gmail.com \\ Bárbara Regina Alvarez \\ ORCID: https://orcid.org/0000-0003-3433-516X \\ Universidade do Extremo Sul Catarinense, Brasil \\ E-mail:bra@unesc.net
}

\begin{abstract}
Resumo
O Beach Tennis atualmente é uma prática que vem crescendo muito no mundo inteiro, especialmente pela facilidade com que uma pessoa aprende a jogar e pela diversão que ele proporciona. O Beach Tennis é um esporte praticado em quadra de areia, com regras e execução de movimentos semelhantes às do tênis. Com a recém chegada do esporte nas quadras de Criciúma, este estudo tem como objetivo analisar o perfil dos praticantes de beach tennis no município, tratando-se de uma pesquisa de abordagem quantitativa descritiva para análise dos dados que foram coletados através de um questionário com perguntas fechadas que foi elaborado com o objetivo de identificar o perfil dos praticantes. Participaram da pesquisa 81 praticantes voluntários. Concluiu-se que o público que pratica o esporte na cidade de Criciúma é composto por sua maioria no gênero feminino 75,3\%, idades entre 25 a 35 anos. Em nível de escolaridade $85,2 \%$ possuem ensino superior e $60,5 \%$ são casados. Os praticantes por sua maioria são funcionários em empresa privada, empresários e profissionais liberais onde possuem uma renda mensal acima de 3.500 reais $(80,20 \%)$ e moram em Criciúma (87,70\%), em uma distância entre 1 a 4 km e todos utilizam automóvel como meio de transporte. Quanto as características dos treinos a maioria treinam de 1 a 3 meses $(48,15 \%)$, praticam por recreação $(59,26 \%)$, pelo fato da maioria ser iniciante no beach tennis eles participam da categoria D (44,83\%) nos campeonatos e também 75,30\% possuem raquete própria e não treinam somente o beach tennis.
\end{abstract}

Palavras-chave: Beach Tennis; Perfil; Praticantes; Questionário; Análise.

\begin{abstract}
Beach Tennis is currently a practice that has been growing a lot all over the world, especially because of the ease that a person learns to play and for the fun that it provides. Beach Tennis is a sport played on a sand court, with rules and execution of movements similar to those of tennis. With the recent arrival of the sport in the courts of Criciúma, this study aims to analyze the profile of beach tennis players in the city. descriptive quantitative research approach to analyze the data that were collected through a questionnaire with closed questions that was prepared in order to identify the profile of practitioners. Participated in the research 81 voluntary practitioners. It was concluded that the public who practices the sport in the city of Criciúma is composed mostly of female gender $75.3 \%$, ages between 25 and 35 years. In level of education, $85.2 \%$ have higher education and $60.5 \%$ are married. Most of the practitioners are employees of private companies, businessmen, and liberal professionals have a monthly income above 3,500 reais (80.20\%) and live in Criciúma (87.70\%), in a distance between 1 to $4 \mathrm{~km}$ and all use a car as means of transportation. As for the characteristics of the training sessions, most train for 1 to 3 months (48.15\%), they practice for recreation (59.26\%), and because most of them are beginners in beach tennis they participate in category $\mathrm{D}(44.83 \%)$ in the championships and also $75.30 \%$ have their own racket and do not train only in beach tennis.
\end{abstract}

Keywords: Beach Tennis; Profile; Practitioners; Quiz; Analysis.

\section{Resumen}

El tenis de playa es actualmente una práctica que ha crecido mucho en todo el mundo, especialmente por la facilidad que una persona aprende a jugar y por la diversión que proporciona. El tenis de playa es un deporte que se practica en una pista de arena, con reglas y ejecución de movimientos similares a los del tenis. Con la reciente llegada del deporte a los tribunales de Criciúma, este estudio tiene como objetivo analizar el perfil de los practicantes de tenis de playa en la ciudad, siendo una investigación de enfoque cuantitativo descriptivo para el análisis de los datos que se recogieron a través de un cuestionario con preguntas cerradas que se preparó para identificar el perfil de los practicantes. Participó en la investigación 81 practicantes voluntarios. Se concluyó que el público que practica el deporte en la ciudad de Criciúma está compuesto por su mayoría em sexo femenino $75,3 \%$, edades entre 25 y 35 años. En cuanto al nivel de 
escolaridad, el 85,2\% tiene estudios superiores y el 60,5\% es casado. La mayoría de los practicantes son empleados de empresas privadas, empresarios y profesionales donde tienen una renta mensual superior a $\mathrm{R} \$ 3.500(80,20 \%)$ y viven en Criciúma (87,70\%), a una distancia de 1 a $4 \mathrm{~km}$ y todos ellos utilizan el coche como medio de transporte. En cuanto a las características de la formación, la mayoría entrena de 1 a 3 meses $(48,15 \%)$, practicar por recreación (59,26\%), porque la mayoría son principiantes en el tenis de playa participan en la categoría D $(44,83 \%)$ en los campeonatos y también el 75,30\% tiene su propia raqueta y no entrena sólo tenis de playa.

Palabras clave: Tenis de playa; Perfil; Practicantes; Examen; Analisis.

\section{Introdução}

Praticar atividades físicas durante a pandemia se tornou um grande desafio. Na busca por uma modalidade esportiva sem contato físico e com respeito ao distanciamento, o beach tennis ganhou um expressivo número de adeptos ao longo do último ano, uma vez que o esporte é uma fonte de bem-estar físico e mental e podem ser praticados por pessoas de todas as idades. Além disso, auxiliam na coordenação motora, condicionamento físico e podem ajudar a emagrecer. O Beach Tennis conquistou os brasileiros por ser ao ar livre o que traz mais prazer e motivação, por ser praticado na areia, a movimentação torna-se mais pesada e, por isso, exige mais esforço nas pernas. É um esporte fácil de aprender, dinâmico e que favorece a socialização já que é feito em um ambiente mais descontraído. A modalidade apresenta muita semelhança ao tênis, frescobol e vôlei de praia. A sua origem exata ainda é desconhecida, mas de acordo com a Confederação Brasileira de Tênis (2021) acredita-se que teve início nas praias italianas em meados da década de 90, sem qualquer tipo de regra, quadra, raquete ou bola específica. Foi somente em 1996 que o Beach Tennis começou a se desenvolver, onde então foi estabelecido regras e dimensões da quadra, neste momento ele deixou de ser praticado somente como lazer e diversão e se torna realmente o esporte.

Segundo informações encontradas no site de Cortez, J. (2008), uma das pioneiras do Beach Tennis em nosso país, este esporte apareceu no Brasil em 2008, por iniciativa de Leopoldo Correa, Adão Chagas e Pedro Dias, que foram os primeiros a ensiná-lo na praia de Ipanema.

Santini e Mingozzi (2017) defendem que a modalidade é acessível a todas as faixas etárias. Contudo, destacam a importância do acompanhamento de um especialista na iniciação esportiva, para que se aprenda bem a técnica e os elementos estratégicos do jogo. Nas palavras de Quarantini (2010), o BT desenvolve algumas capacidades físicas, tais como a força e a resistência muscular, além da melhora no sistema cardiorrespiratório. A prática na areia e o manuseio da raquete promove o ganho de diversas habilidades motoras e coordenativas, já que a velocidade do jogo exige agilidade dos praticantes e boa técnica no contato da raquete com a bola (Mucchi, 2013).

Silva (2021) em sua pesquisa desenvolvido na cidade de Santos-SP avaliou as capacidades físicas, composição corporal e qualidade de vida de mulheres adultas praticantes e não praticantes de beach tennis. Amostra composta por 16 mulheres entre 20 e 50 anos. E como resultado obtiveram diferenças significativas em relação as variáveis de massa corporal, IMC, média geral de qualidade de vida, estado geral de saúde, gordura relativa (total e segmentada), massa muscular relativa, massa muscular absoluta de perna esquerda, quantidade de água corporal, taxa metabólica basal e gasto energético semanal.

Apesar do esporte parecer inofensivo, Neumann (2021) identificou em seu estudo que entre os seus 202 participantes de questionários eletrônico apresentaram mais de uma lesão decorrente do beach tennis. A maioria das lesões aconteceram no membro superior, principalmente no ombro e cotovelo. No entanto, também foi registrado um número significativo de lesões no membro inferior, sendo o joelho e o tornozelo os mais acometidos neste segmento.

Hoje, o Beach Tennis tem inúmeros eventos em seu calendário, como o Campeonato Mundial de Equipes, o Campeonato Europeu, o Campeonato Mundial Individual, bem como todas as competições internacionais e nacionais. Os locais onde há um maior número de praticantes estão Rio de Janeiro, Fortaleza, Santos, Vitória, Florianópolis, Porto Alegre, Mogi das Cruzes, Guarujá entre outros. Atualmente, segundo a Federação de Beach Tennis do Estado da Bahia (FBTEB, 2011) existem, aproximadamente, 500 mil jogadores espalhados pelo mundo. Os países onde encontramos Beach Tennis são: Itália, Brasil, 
França, Espanha, Rússia, Estados Unidos, Japão, Portugal, República Tcheca, Bermudas, Holanda, Aruba, Austrália, Curaçao e Chile. O maior evento de Beach Tennis da atualidade é o "ITF Beach Tennis World Championships" realizado em Roma, com jogadores de várias nacionalidades, que ocorre todo ano no mês de maio.

Já as regras do Beach Tennis foram criadas pela International Tennis Federation (ITF), onde algumas repetem o que é utilizado no tênis de quadra, porem temos importantes diferenças, principalmente em relação aos pontos e ao saque. As bolas utilizadas no esporte devem ser aprovadas pela ITF e devem seguir o manual de normas técnicas ITF Approved Tennis Balls, Classified Surfaces \& Recognised Courts a guide to products \& test methods (Federação Internacional de Tênis, 2016). As quadras de Beach Tennis, por regra, são retangulares e possuem 16 metros de comprimento. A largura dela é variada, sendo que em jogos de duplas é de 8 metros e nos jogos de simples ela fica em 4,5 metros de largura. A pontuação no Beach Tennis segue a escala de 15, 30, 40 e game. Os jogos de simples são disputados em melhor de 6 games e as de duplas em melhor de 9 games, com diferença de 2 games. As principais categorias são: simples feminina, simples masculina, dupla feminina, dupla masculina e dupla mista. No Brasil, os torneios geralmente incluem as categorias: Profissional, A, B, C, D (iniciante) Master +45 e infantil (Westrupp, 2021).

De acordo com Albertini, L. (2021) Criciúma é uma cidade da Região Sul, localizado no estado de Santa Catarina, na mesorregião do Sul Catarinense. Tem 219.393 habitantes, sendo a principal cidade da Região Metropolitana Carbonífera, a mais populosa do Sul Catarinense e a quinta do estado de Santa Catarina. Em Criciúma o beach tennis é um esporte que está em crescente evolução, cada dia aumenta a expectativa do público em querer aprender esse novo esporte para lazer e também competir, na cidade já aconteceram alguns campeonatos e houve um grande número de participantes.

Mesmo com o grande número de praticantes muitos dos cidadãos não tem disponibilidade para ir todos os dias às praias, seja pela distância ou por conta do horário comercial, por exemplo. Com isso uma nova tendência surgiu em Criciúma, aumentou os números de quadras de areias em Complexos Esportivos. Sendo o principal objetivo a areia das praias mais perto da sociedade, de forma que os praticantes e interessados pelos esportes tenham a oportunidade de alugar horários para jogar ou horários para treinar com profissionais capacitados. De acordo com Castro (2021) o amor pela areia não é novidade para esportistas, mas com a situação atual da pandemia o potencial brilhou especialmente para as quadras de areia que são em lugares abertos, e deu abertura principalmente para o crescimento do beach tennis pois a modalidade não possui contato físico. Apesar da grande concorrência, com o aumento da demanda e procura da população por horários disponíveis e nos horários que mais lhe convém, percebe-se uma boa oportunidade na inserção do negócio na cidade, alinhado com uma análise mais aprofundada sobre fatores como perfil socioeconômico e características das aulas de beach tennis.

O objetivo desta pesquisa foi analisar o perfil dos praticantes de Beach Tennis no município de Criciúma. Além de tudo, o presente trabalho contribui academicamente uma vez que permite que os autores do mesmo apliquem de forma sistêmica e integrada os conhecimentos adquiridos com essa pesquisa, além desse levantamento pretende-se contribuir para os profissionais dessa área com o objetivo de aumentar os praticantes de beach tennis no município.

\section{Metodologia}

Trata-se de um estudo observacional de abordagem quantitativa descritiva de coleta de dados primário. Segundo Knechtel (2014), a pesquisa quantitativa é uma modalidade de pesquisa que atua sobre um problema humano ou social, é baseada no teste de uma teoria e composta por variáveis quantificadas em números, as quais são analisadas de modo estatístico, com o objetivo de determinar se as generalizações previstas na teoria se sustentam ou não. A partir dos objetivos explicitados na introdução, o instrumento que utilizamos para coleta de dados foi um questionário fechado. Para a aplicação do questionário primeiramente foi entrado em contato com todos os professores que possuem alunos de Beach Tennis em Criciúma, logo após os professores mandaram em seus grupos de whats app o link do Google Forms e solicitaram para que seus alunos ficassem à 
vontade para responde-lo.

O questionário estruturado é composto por 19 questões onde foi gerado por meio de uma ferramenta gratuita oferecida pelo Google: o Google Forms. Para a realização da pesquisa o questionário ficou disponível para preenchimento no site do Google Forms no período de 31/08/2021 a 09/09/2021 no endereço web a seguir: https://forms.gle/1WWCZ1jcwK1fQcRd9. Para a aplicação do questionário, realizamos primeiramente os procedimentos legais e éticos da pesquisa que envolveram a apresentação de seus objetivos, o pedido formal para sua realização aos professores, bem como o aceite dos alunos para o estudo por meio do termo de Consentimento Livre e Esclarecido (TCLE). Ao final obtivemos 81 respostas e o próprio Google Forms manteve um resumo das respostas em modelo gráfico, a fim de melhorar a visualização.

\section{Resultados e Discussão}

A seguir são apresentadas todas as informações coletadas na presente pesquisa, relativas ao perfil dos praticantes de Beach Tennis no município de Criciúma. Observa-se que o questionário aplicado aos alunos foi elaborado em conformidade com os indicadores sociais utilizados para realizações de pesquisas do IBGE relacionadas aos fatores socioeconômicos. Durante o preenchimento do questionário, o aluno pôde somente optar por uma alternativa em cada questão.

Características dos participantes em relação ao gênero, faixa etária, escolaridade, estado civil, conforme podemos verificar no Quadro 1.

Quadro 1 - Características Pessoais

\begin{tabular}{|c|c|c|c|}
\hline \multirow{4}{*}{ GÊNERO } & VARIÁVEL & QUANTIDADE & $\%$ \\
\hline & Feminino & 61 & $75,30 \%$ \\
\hline & Masculino & 20 & $24,70 \%$ \\
\hline & TOTAL & 81 & $100 \%$ \\
\hline \multirow{7}{*}{ FAIXA ETÁRIA } & Menos de 18 anos & - & - \\
\hline & 18 a 24 anos & 7 & $9 \%$ \\
\hline & 25 a 35 anos & 36 & $44 \%$ \\
\hline & 36 a 46 anos & 24 & $30 \%$ \\
\hline & 47 a 57 anos & 10 & $12 \%$ \\
\hline & Mais de 58 anos & 4 & $5 \%$ \\
\hline & TOTAL & 81 & $100 \%$ \\
\hline \multirow{7}{*}{ ESCOLARIDADE } & Ensino Fundamental Incompleto & - & - \\
\hline & Ensino Fundamental Completo & 1 & $1,2 \%$ \\
\hline & Ensino Médio Incompleto & 1 & $1,2 \%$ \\
\hline & Ensino Médio Completo & 6 & $7,4 \%$ \\
\hline & Superior & 69 & $85,2 \%$ \\
\hline & Mestrado ou Doutorado & 4 & $5 \%$ \\
\hline & TOTAL & 81 & $100 \%$ \\
\hline \multirow{6}{*}{ ESTADO CÍVIL } & Solteiro & 29 & $35,8 \%$ \\
\hline & Casado & 49 & $60,5 \%$ \\
\hline & Viúvo & - & - \\
\hline & Divorciado & 2 & $2,5 \%$ \\
\hline & Outro & 1 & $1,2 \%$ \\
\hline & TOTAL & 81 & $100 \%$ \\
\hline
\end{tabular}

Fonte: Dados da Pesquisa (2021). 
Analisando os dados coletados no Quadro 1, constata-se que o número de praticantes do gênero feminino $(75,3 \%)$ comparado com masculino $(24,7 \%)$ é de maior prevalência, ou seja, tem um número expressivo do público feminino em busca da modalidade, onde as mulheres viram no beach tennis um esporte com clima agradável, sem preconceito e de fácil aprendizagem para praticarem. De acordo com Sampaio (2015) em números divulgados pelo IBGE no Brasil, 61,3 milhões de pessoas de 15 anos ou mais de idade - o equivalente a 37,9\% do total de 161,8 milhões de pessoas nessa faixa etária, praticavam algum tipo de esporte ou atividade física, dos quais 53,9\% eram homens e 46,1\%, mulheres. Quando comparado a faixa etária percebemos que $44 \%$ são referente as idades de 25 a 35 anos e $30 \%$ as idades de 36 a 46 anos. A predominância de adultos no esporte sempre foi algo recorrente, mesmo com todas as atividades diárias e trabalhos cansativos a grande população adulta reserva um horário do seu dia para a prática de alguma atividade física ou esportiva. Apesar da grande porcentagem de adultos praticantes também temos $17 \%$ de pessoas acima de 47 anos. Segundo Cabral (2014) o público idoso está cada vez mais presente em práticas esportivas. Com o aumento da expectativa de vida decorrente do avanço da medicina e com a vida mais ativa a possibilidade de os mais velhos serem inseridos aos esportes aumenta consideravelmente.

Conforme a escolaridade mais da metade dos entrevistados possuem nível superior $85,2 \%$ e 7,4\% possuem ensino médio completo, apresentando um nível de formação elevado. De acordo com Nascimento et. al (2012) em sua pesquisa sobre o perfil de praticantes de beach tennis filiados à federação de tênis do estado do rio de janeiro também apresentou na situação de amostra em relação ao estudo formal no momento da entrevista e demonstra que $70 \%$ dos entrevistados estudavam, sendo que sua maior porcentagem foi de $48 \%$ para ensino superior.

Com isso também percebemos a situação conjugal, ainda que a maioria seja de casados 60,5\%, o percentual de solteiros chega a cerca de 35,8\%. Segundo Sillva, G (2021), a dinâmica do jogo ajuda na socialização, pois pode ser jogado em forma individual ou em duplas pois as pessoas se divertem e se conhecem. Como o esporte é praticado em duplas, então permite este convívio social com quem pratica, principalmente com os casados pois podem jogar juntos e não um contra o outro, não entrando em um clima de competição. 
Quadro 2 - Perfil Socioeconômico.

\begin{tabular}{|c|c|c|c|}
\hline \multirow{10}{*}{ OCUPAÇÃO } & VARIÁVEL & QUANTIDADE & $\%$ \\
\hline & Estudante & 9 & $11,10 \%$ \\
\hline & Funcionário Público & 8 & $9,90 \%$ \\
\hline & Funcionário em empresa privada & 21 & $25,90 \%$ \\
\hline & Empresário & 20 & $24,70 \%$ \\
\hline & Profissional Liberal & 13 & $16,05 \%$ \\
\hline & Desempregado & - & - \\
\hline & Aposentado & 4 & $4,94 \%$ \\
\hline & Outro & 6 & $7,41 \%$ \\
\hline & TOTAL & 81 & $100 \%$ \\
\hline \multirow{7}{*}{ ONDE MORA } & Criciúma & 71 & $87,70 \%$ \\
\hline & Forquilhinha & - & - \\
\hline & Içara & 7 & $8,60 \%$ \\
\hline & Cocal do Sul & - & - \\
\hline & Urussanga & - & - \\
\hline & Outro & 3 & $3,70 \%$ \\
\hline & TOTAL & 81 & $100 \%$ \\
\hline \multirow{5}{*}{ DISTÂNCIA } & Até 1 km & 6 & $7,40 \%$ \\
\hline & De 1 a $4 \mathrm{~km}$ & 36 & $44,40 \%$ \\
\hline & De 5 a $10 \mathrm{~km}$ & 28 & $34,60 \%$ \\
\hline & Mais de $10 \mathrm{~km}$ & 11 & $13,60 \%$ \\
\hline & TOTAL & 81 & $100 \%$ \\
\hline \multirow{5}{*}{ TRANSPORTE } & Moto & - & - \\
\hline & Automóvel & 81 & $100 \%$ \\
\hline & Bicicleta & - & - \\
\hline & Outro & - & - \\
\hline & TOTAL & 81 & $100 \%$ \\
\hline \multirow{5}{*}{ RENDA BRUTA MENSAL } & Até $\mathrm{R} \$ 1.100,00$ reais & 3 & $3,70 \%$ \\
\hline & De $\mathrm{R} \$ 1.100,00$ a $\mathrm{R} \$ 2.500$ reais & 2 & $2,50 \%$ \\
\hline & De $\mathrm{R} \$ 2.500$ reais a 3.500 reais & 11 & $13,60 \%$ \\
\hline & Mais de $\mathrm{R} \$ 3.500$ reais & 65 & $80,20 \%$ \\
\hline & TOTAL & 81 & $100 \%$ \\
\hline
\end{tabular}

Fonte: Dados da Pesquisa (2021)

O Quadro 2 contém os dados referentes ao perfil socioeconômico dos participantes da pesquisa, envolvendo a ocupação, local que mora, distância, transporte e renda mensal. Dos participantes que responderam em relação à ocupação, observa-se que $25,9 \%$ são funcionários de empresa privada e 24,7\% empresários e no que se refere à renda mensal observa-se que 80,2\% recebem mais de 3.500 reais por mês.

De acordo com Capozzoli (2010) os grupos de baixa renda parece haver evidências de que a inatividade pode estar sendo reforçada em função do desconhecimento acerca dos benefícios positivos que a prática pode proporcionar, sendo assim indivíduos com renda e grau de instrução mais elevado parecem ter mais probabilidade de serem fisicamente ativos. Pode-se relacionar essa afirmação do autor com os dados obtidos até então na presente pesquisa, que apresenta indivíduos com elevado nível de escolaridade, tendo como ocupação; empregados de empresas privadas, empresários e profissionais liberais assim necessitando de um grau elevado de ensino para ocupar os respectivos cargos. 
Quando observamos onde os participantes moram, distância do local de treinamento e o seu meio de transporte percebemos que 87,7\% moram em Criciúma e 12,3\% moram em outra cidade, 100\% deles utilizam automóveis como meio de transporte e 44,4\% são participantes que moram de 1 a 4 km do local e 34,6\% de 5 a $10 \mathrm{~km}$. Ou seja, percebemos que os participantes estão optando por locais que são perto de sua casa ou de seu local de trabalho, mas em relação ao meio de transporte estão utilizando carros mesmo em distâncias muitas pequenas. Marques (2015) afirma que enquanto tivermos mais carros nas estradas, maior congestionamento, poluição, consumo de combustível e emissões de gases de efeito estufa teremos. Se a quantidade de viagens de carro acompanhar o mesmo ritmo do crescimento da população, os ganhos com a eficiência do combustível e tecnologias mais limpas serão anulados por velocidades menores e os motoristas continuarão presos nos congestionamentos. Os praticantes poderiam optar por estar indo de bicicleta ou até mesmo a pé para o local de treino pois serviria como um aquecimento para o corpo aumentando a frequência cardiorrespiratória e levando mais sangue para os músculos preparando o corpo para a atividade mais intensa que irá ser realizada depois.

Quadro 3 - Características do Treino.

\begin{tabular}{|c|c|c|c|}
\hline \multirow{9}{*}{ TEMPO DE PRÁTICA } & VARIÁVEL & QUANTIDADE & $\%$ \\
\hline & 1 mês a 3 meses & 39 & $48,15 \%$ \\
\hline & 4 meses a 6 meses & 12 & $14,81 \%$ \\
\hline & 7 meses a 9 meses & 9 & $11,11 \%$ \\
\hline & 10 meses a 12 meses & 8 & $9,88 \%$ \\
\hline & Mais de 1 ano & 10 & $12,35 \%$ \\
\hline & Mais de 2 anos & 2 & $2,47 \%$ \\
\hline & Mais de 3 anos & 1 & $1,23 \%$ \\
\hline & TOTAL & 81 & $100 \%$ \\
\hline \multirow{8}{*}{ TREINOS POR SEMANA } & 1 vez por semana & 24 & $29,63 \%$ \\
\hline & 2 vezes por semana & 30 & $37,04 \%$ \\
\hline & 3 vezes por semana & 18 & $22,22 \%$ \\
\hline & 4 vezes por semana & 6 & $7,41 \%$ \\
\hline & 5 vezes por semana & 3 & $3,70 \%$ \\
\hline & 6 vezes por semana & - & - \\
\hline & 7 vezes por semana & - & - \\
\hline & TOTAL & 81 & $100,00 \%$ \\
\hline \multirow{4}{*}{ PERÍDO DE TREINO } & Manhã & 17 & $21 \%$ \\
\hline & Tarde & 15 & $18,50 \%$ \\
\hline & Noite & 49 & $60,50 \%$ \\
\hline & TOTAL & 81 & $100 \%$ \\
\hline \multirow{4}{*}{$\begin{array}{l}\text { MOTIVO DA PRÁTICA DE BEACH } \\
\text { TENNIS }\end{array}$} & Recreação & 48 & $59,26 \%$ \\
\hline & Competitivo & 17 & $20,99 \%$ \\
\hline & Condicionamento Físico & 16 & $19,75 \%$ \\
\hline & TOTAL & 81 & $100 \%$ \\
\hline \multirow{6}{*}{$\begin{array}{l}\text { CATEGORIA DE QUEM JOGA POR } \\
\text { COMPETIÇÃO * }\end{array}$} & A & 1 & $3,45 \%$ \\
\hline & $\mathrm{B}$ & 3 & $10,34 \%$ \\
\hline & $\mathrm{C}$ & 12 & $41,38 \%$ \\
\hline & $\mathrm{D}$ (Iniciante) & 13 & $44,83 \%$ \\
\hline & Profissional & - & - \\
\hline & Master +45 & - & - \\
\hline
\end{tabular}




\begin{tabular}{|c|c|c|c|}
\hline & Infantil & - & - \\
\hline & TOTAL & 29 & $100 \%$ \\
\hline \multirow{3}{*}{$\begin{array}{l}\text { NÃO ESCOLHEU COMPETITIVO, } \\
\text { MAS TEM INTERESSE DE } \\
\text { PARTICIPAR DE CAMPEONATOS * }\end{array}$} & Sim & 41 & $67,20 \%$ \\
\hline & Não & 20 & $32,80 \%$ \\
\hline & TOTAL & 61 & $100 \%$ \\
\hline \multirow{3}{*}{$\begin{array}{l}\text { PARTICIPOU DE ALGUM } \\
\text { CAMPEONATO }\end{array}$} & Sim & 36 & $44,40 \%$ \\
\hline & Não & 45 & $55,60 \%$ \\
\hline & TOTAL & 81 & $100 \%$ \\
\hline \multirow{3}{*}{ POSSUI RAQUETE PRÓPRIA } & Sim & 61 & $75,30 \%$ \\
\hline & Não & 20 & $24,70 \%$ \\
\hline & TOTAL & 81 & $100 \%$ \\
\hline \multirow{3}{*}{ PRÁTICA SOMENTE BEACH TENNIS } & Sim & 21 & $25,90 \%$ \\
\hline & Não & 60 & $74,10 \%$ \\
\hline & Total & 81 & $100 \%$ \\
\hline
\end{tabular}

*ñ é obrigatório responder essa pergunta. Fonte: Autores.

Com os dados descritos no quadro 3 referentes as características do treino de beach tennis no municio de Criciúma, logo na primeira pergunta já analisamos que a grande parte dos praticantes treinam entre 1 a 3 meses $(48,15 \%)$ e 2 vezes por semana (37,04\%). Afirmando assim que a modalidade ainda é muito nova na região, mas que está em crescente aumento. Segundo Prata,

G. (2019) em seu livro de Capacitação de Beach Tennis, no começo do esporte no Brasil houve muita desconfiança sobre o potencial de crescimento exponencial, o beach tennis se mostra como um negócio promissor e vem atraindo cada vez mais investidores. Dos praticantes 60,5\% preferem jogar a noite, e jogam por recreação 59,26\%. Com bases em números divulgados em 2015 pelo IBGE no Brasil, em um levantamento de 11,2 milhões de pessoas apontaram como principal motivo para a prática de algum esporte a necessidade de relaxar ou se divertir, sendo tal justificativa mais comum entre os homens (37,8\%) do que entre as mulheres (13,5\%). De acordo com Balbinotti et al (2015) o prazer está relacionado à sensação de bem estar, de diversão e à satisfação que a prática regular de atividade física e/ou esporte proporciona.

Entre aqueles que responderam que jogam por competição, fazem parte da categoria D 44,83\% e 41,38\% na categoria C. Daqueles que não escolherem competitivo, 67,2\% pretendem participar de campeonato e 55,6\% nunca participaram de campeonatos. Pelo fato do esporte ser novo e das pessoas começarem a pratica-lo recentemente a sua grande maioria disputa nas categorias iniciais, possuem raquetes próprias (75,3\%), mesmo quem nunca participou de nenhum campeonato tem planos futuros para participar. Dos participantes $74,1 \%$ responderão que não praticam somente o beach tennis. Para Nahas, (2006) os estudos realizados têm demonstrado evidências de que as pessoas que possuem um estilo de vida ativo tendem a ter uma auto estima e uma percepção de bem-estar psicológico positivo.

\section{Considerações Finais}

A presente pesquisa identificou que os praticantes de Beach Tennis no munícipio de Criciúma, são de maior prevalência do gênero feminino, na faixa etária de 25 a 35 anos, casados, possuem uma renda mensal bruta alta devido principalmente ao fator de sua escolaridade ser de nível elevado e também da sua ocupação, onde a grande porcentagem são de funcionários de empresas privadas e empresários. Nas características dos treinos percebemos que o grande público prefere treinar a noite, 2 vezes por semana, no qual os participantes de campeonatos fazem parte das categorias D e C. Também percebemos a grande procura de campeonatos, esperamos que agora com o verão aumente consideravelmente o número de campeonatos.

Uma sugestão para os donos de complexos esportivos de Criciúma é fazer mais treinos no turno da noite, já que a pesquisa relatou que os praticantes preferem treinar nesse turno provavelmente por sua rotina. Dar ênfase também ao 
estacionamento pois $100 \%$ dos praticantes se locomovem para os treinos de carro, e para isso precisa-se de um estacionamento amplo. Vimos a necessidade de mais complexos esportivos em Criciúma e também em cidades próximas. Os complexos também poderiam estar tendo raquetes para aluguel para alunos que ainda não têm ou que acabaram esquecendo. Quando as turmas estiverem fechadas poderiam ser aplicadas alguma anamnese para os alunos para identificar o seu principal objetivo com os treinos. Percebemos também uma necessidade de mais professores e cursos de capacitação para professores de beach tennis.

A pesquisa revelou dados fundamentais para apliquem de forma sistêmica e integrada os conhecimentos adquiridos, através desse levantamento pretende-se contribuir para os profissionais dessa área. Assim, acreditamos que trabalhos futuros devem ser realizados sobre várias áreas do beach tennis, pois a modalidade vem crescimento muito e precisamos de mais estudos. Embora o beach tennis seja um esporte novo no país, a sua grande ascensão é notável e provável que continuará por muitos anos, devemos dar o devido valor para a modalidade e também para os profissionais qualificados para o esporte.

\section{Referências}

Albertini, L. (2021). Instituto brasileiro de geografia e estatística. Criciúma.

Balbinotti, M. A. A. (2015). Perfis motivacionais de corredores de rua com diferentes tempos de prática. Revista Brasileira de Ciência do Esporte.

Cabral, J. (2014). Tênis na terceira idade. Revista Tênis.

Capozzoli, C. J. (2010) Motivação à prática regular de atividade física: um estudo com praticantes em academias de ginástica de Porto Alegre.

Castro, A. F. (2021). De futevôlei a beach tênis: entenda o boom dos esportes pé na areia.

Confederação Brasileira de Tênis. (2021). Regras de Tênis de Praia.

Cortez, J (2008). História.

Federação Internacional de Tênis. (2016). ITF. Approved Tennis Balls, Classified Surfaces \& Recognised Courts a guide to products \& test methods.

Knechtel, M. R. (2014). Metodologia da pesquisa em educação: uma abordagem teórico-prática dialogada.

Marques, R. (2015). Desestímulo ao uso do automovel.

Mucchi, E. (2013). On the sweet spot estimation in beach tennis rackets. Measurement, 46(4), 1399- 1410.

Nascimento, F, (2012). O perfil de praticantes de beach tennis filiados à federação de tênis do estado do rio de janeiro - CORE Reader

Nahas, M. V. (2006). Atividade física, saúde e qualidade de vida: conceitos e sugestões para um estilo de vida ativo.

Neumann, A. (2021). A incidência de lesões em atletas praticantes de beach tennis.

Prata, G., Rodrigues, N., Mingozzi, A., \&Russo, Plácido. (2019). Capacitação de Beach Tennis.

Quarantini, M. I. (2010). l manuale del Beach Tennis.

Sampaio, K. (2015). Instituto brasileiro de geografia e estatística: 100 milhões de pessoas com 15 anos ou mais não praticam esporte no Brasil. Agência Brasil.

Santini, J., Mingozzi, A. (2017). Beach Tennis: um esporte em ascensão.

Silva, E. A. (2021). Composição corporal, capacidades físicas e qualidade de vida de praticantes e não praticantes de beach tennis do sexo feminino.

Sillva, G. (2021). 5 benefícios para começar a treinar o tênis de praia.

Westrupp, R. B. (2021). Beach Tennis: Confederação Brasileira de Tênis. 